\title{
Effect of methotrexate and doxorubicin cumulative doses on superoxide dismutase levels in childhood acute lymphoblastic leukemia
}

\author{
Khalida Fetriyani Ningsih, Sri Mulatsih, Sasmito Nugroho
}

\begin{abstract}
Background Acute lymphoblastic leukemia (ALL) is the most common malignancy in children. Chemotherapeutic drugs for ALL such as methotrexate (Mtx) and doxorubicin produce reactive oxygen species (ROS), a type of free radical. The ROS can reduce levels of antioxidants in the body, including superoxide dismutase (SOD). Decreased SOD levels can cause DNA, lipid, and protein damage, which in turn may lead to adverse effects and treatment failure.

Objective To determine the effect of Mtx and doxorubicin cumulative doses on SOD levels in children with ALL.

Methods We conducted a retrospective cohort study in children with ALL who underwent chemotherapy in Dr. Sardjito Hospital in October 2011 who had completed the induction phase. Risk factors for decreased SOD levels were analyzed by Cox regression and hazard ratio, with a significant level of $\mathrm{P}<0.05$.

Results Of 40 patients enrolled, Mtx $\geq 3000 \mathrm{mg} / \mathrm{m}^{2}$ significantly decreased SOD levels (HR 9.959; 95\%CI 2.819 to 35.183; $\mathrm{P}=0.001)$. However, doxorubicin $\geq 90 \mathrm{mg} / \mathrm{m}^{2}$ did not significantly decrease SOD levels (HR 0.59 95\%CI 0.194 to 1.765 ; $\mathrm{P}=0.34$ ). Conclusion Methotrexate is associated with decreased SOD levels in children with ALL. However, doxorubicin is not associated with decreased SOD levels in the same patient population. [Paediatr Indones. 2015;55:239-42].
\end{abstract}

Keywords: acute lymphoblastic leukemia, methotrexate, doxorubicin, superoxide dismutase
A cute lymphoblastic leukemia (ALL) is the most common malignancy in children, comprising nearly one-third of childhood malignancies. Every year more children are diagnosed with ALL. In Dr. Sardjito Hospital, Yogyakarta, from 2000 to 2004, 486 children with cancer were treated. Of these children, $35 \%$ had ALL and $13 \%$ had acute myeloblastic leukemia (AML). ${ }^{1}$

The primary treatment for ALL is chemotherapy. Although chemotherapy has been shown to be successful, the formation of free radicals and their toxic effects has significantly contributed to mortality. ${ }^{2}$ In Dr. Sardjito Hospital, mortality due to childhood ALL remains high. The mortality rate was $8.8 \%$ for those at standard risk and $17.7 \%$ for those at high risk in the induction phase. ${ }^{1}$ The detrimental effect of free radicals in the induction phase has been questioned in the past. Chemotherapy such as methotrexate (Mtx) and doxorubicin can produce reactive oxygen species (ROS), a type of free radical. ${ }^{3}$ The ROS can be determined by examining superoxide dismutase (SOD) levels. ${ }^{4}$ Longer Mtx treatment and greater cumulative doses of this

From the Department of Child Health, Gadjah Mada University Medical School/Dr. Sardjito Hospital, Yogyakarta, Indonesia

Reprint requests to: Khalida Fetriyani Ningsih, MD, Department of Child Health, Gadjah Mada University Medical School/Dr. Sardjito Hospital, Jalan Kesehatan no.1, Yogyakarta, Indonesia. Tel 08125045746, Email: dr.helida@gmail.com. 
drug tends to decrease SOD levels. ${ }^{5}$ Similar results were reported for daunorubicin chemotherapy. ${ }^{6}$ Patients who received high-dose chemotherapy had a significant decrease in plasma concentrations of antioxidant enzymes including SOD due to the formation of free radicals from chemotherapy. ${ }^{6,7}$ Previous studies have examined the relationship between chemotherapy duration in leukemia and SOD levels, without specifying the chemotherapy doses. We aimed to assess the effect of cumulative doses of doxorubicin and Mtx on SOD levels.

\section{Methods}

We conducted a retrospective cohort study in October 2011 at the Department of Child Health, Gadjah Mada University Medical School/Dr. Sardjito Hospital, Yogyakarta.

Sampling was conducted consecutively. Inclusion criteria were ALL patients aged 0-18 years who had completed the induction phase of chemotherapy and whose parents provided informed consent. We excluded patients with incomplete medical records. The SOD levels were assessed using the SOD Cayman chemical kit by Elisa method at the Biochemical Laboratory, Gadjah Mada University Medical School. The cut-off points for SOD was 7.3 $\mathrm{mg} / \mathrm{dL}$. We used SOD $<7.3 \mathrm{mg} / \mathrm{dL}$ as low SOD, dan $\mathrm{SOD} \geq 7.3 \mathrm{mg} / \mathrm{dL}$ as high SOD. Cumulative doses of Mtx and doxorubicin were calculated from data in the medical records from the initiation of chemotherapy until the examination of SOD levels. This study was approved by Ethics Committee of Gadjah Mada University Medical School.

We analyzed data using Cox regression to calculate hazard ratios for the effects of Mtx and doxorubicin cumulative doses on decreased SOD levels. A P values of $<0.05$ were considered to be statistically significant. Data analyses were performed using SPSS version 17.0 for Windows.

\section{Results}

In October 2011 at Dr. Sardjito Hospital, there were 59 ALL patients who had completed the induction phase. We recruited 40 patients into the study, as 17 patients refused participation and 2 patients had

Table 1. Baseline characteristics of subjects

\begin{tabular}{lc}
\hline Characteristics & $(\mathrm{N}=40)$ \\
\hline Mean age (SD), months & $76.9(44.90)$ \\
Maximum & 181 months \\
Minimum & 22 months \\
Gender, $\mathrm{n}(\%)$ & \\
Male & $22(55)$ \\
Female & $18(45)$ \\
Nutritional status, $\mathrm{n}(\%)$ & \\
Severely wasted & 0 \\
Wasted & $4(10)$ \\
Overweight & $3(7.5)$ \\
Good & $33(82.5)$ \\
Passive smoking, $\mathrm{n}(\%)$ & \\
No & $17(42.5)$ \\
Yes & $23(57.5)$ \\
Cigarette exposure & \\
Maximum number of cigarettes/day & 20 \\
Minimum number of cigarettes/day & 5 \\
\hline
\end{tabular}

Table 2. Effect of Mtx and doxorubicin cumulative doses and other variables on SOD levels (univariate analysis)

\begin{tabular}{|c|c|c|c|c|c|}
\hline Variables & $\begin{array}{l}\text { SOD } \\
\text { Low }\end{array}$ & $\begin{array}{l}\text { SOD } \\
\text { High }\end{array}$ & $\begin{array}{l}\text { Hazard ratio } \\
(\mathrm{HR})\end{array}$ & $95 \% \mathrm{Cl}$ & $P$ value \\
\hline \multicolumn{6}{|c|}{ Mtx cumulative dose, n (\%) } \\
\hline$\geq 3000 \mathrm{mg} / \mathrm{m}^{2}$ & $20(64.5)$ & $11(35.5)$ & 9.96 & 2.819 to 35.183 & $<0.0001$ \\
\hline$<3000 \mathrm{mg} / \mathrm{m}^{2}$ & $2(22.5)$ & 7 (77.8) & & & \\
\hline \multicolumn{6}{|c|}{ Doxorubicin cumulative dose, $\mathrm{n}(\%)$} \\
\hline$\geq 90 \mathrm{mg} / \mathrm{m}^{2}$ & $7(41.2)$ & $10(58.8)$ & 0.59 & 0.194 to 1.765 & 0.34 \\
\hline$<90 \mathrm{mg} / \mathrm{m}^{2}$ & $11(61.1)$ & 7 (38.9) & & & \\
\hline \multicolumn{6}{|c|}{ Passive smoking, n (\%) } \\
\hline Yes & $11(47.8)$ & $12(52.2)$ & 0.96 & 0.370 to 2.496 & 0.93 \\
\hline No & $10(55.0)$ & $7(41.2)$ & & & \\
\hline \multicolumn{6}{|c|}{ Nutritional status, n (\%) } \\
\hline Malnourished & $2(50.0)$ & $2(50.0)$ & 1.06 & 0.094 to 11.864 & 0.96 \\
\hline Overweight & 1 (33.3) & $2(66.7)$ & 0.57 & 0.123 to 2.645 & \\
\hline Good & $15(45.5)$ & $18(54.5)$ & & & \\
\hline
\end{tabular}


incomplete data. Characteristics of subjects are shown in Table 1.

Chemotherapy cut-off point was determined by Cox regression analysis to determine the cumulative dose with the highest hazard ratios by considering significance. Based on the analysis, the cut-off points for Mtx and doxorubicin were $3000 \mathrm{mg} / \mathrm{m}^{2}$ and 90 $\mathrm{mg} / \mathrm{m}^{2}$, respectively.

Univariate analysis was conducted to assess the effect of Mtx or doxorubicin cumulative doses and other variables on SOD levels, using Cox regression to obtain hazard ratios (Table 2).

\section{Discussion}

In our study, the mean age of subjects was 76.95 (SD 44.90) months. This result was consistent with a previous study that showed the mean age of childhood leukemia to be 2 to 15 years with a peak at 2 to 4 years. ${ }^{2}$ Males comprised $55 \%$ and females $45 \%$ of our subjects. Similarly, a previous study reported $55 \%$ of their subjects to be male and $45 \%$ to be female. ${ }^{1}$ Most subjects had good nutritional status (82.5\%), followed by wasted $(10 \%)$, and overweight $(7.5 \%)$. None of the 40 subjects were severely wasted. In contrast, another study reported $62.5 \%$ of their subjects to have malnutrition (wasted and severely wasted) and 31\% to have good nutrition. ${ }^{8}$ For smoking status, $57.5 \%$ of our subjects were passive smokers, exposed to between 5 and 20 cigarettes per day.

Statistical analysis revealed that Mtx cumulative doses of $\geq 3000 \mathrm{mg} / \mathrm{m}^{2}$ had a hazard ratio of $9.96(95 \% \mathrm{CI}$ 2.819 to $35.183 ; \mathrm{P}<0.0001$ ), suggesting that these $\mathrm{Mtx}$ doses decreased SOD levels 9.96 times compared to Mtx doses of $<3000 \mathrm{mg} / \mathrm{m}^{2}$, a statistically significant result. This result was consistent with previous research that longer duration of Mtx treatment decreased the SOD levels in mice, assuming that longer period of Mtx treatment equates to greater cumulative doses $(\mathrm{P}=0.05){ }^{5}$ Asci et al. ${ }^{9}$ also mentioned that Mtx can decrease SOD levels in mice $(\mathrm{P}=0.05)$. Methotrexate stimulates polymorphonuclear (PMN) cells to release $\mathrm{H}_{2} \mathrm{O}_{2}$ and other free radicals, ${ }^{5}$ as well as reduces the effectiveness of antioxidants to fight free radicals and reduces the availability of NADPH used by glutathione-reductase to maintain glutathione levels. Decreased glutathione-reductase causes the PMN to be more sensitive to oxidative stress, and ultimately will decrease the body's antioxidant levels, including SOD. ${ }^{10}$

On the other hand, cumulative doxorubicin doses of $\geq 90 \mathrm{mg} / \mathrm{m}^{2}$ had a hazard ratio of 0.59 (95\%CI 0.194 to $1.765 ; \mathrm{P}=0.34$ ). This finding indicates that these doses led to 0.59 times lower risk of decreased SOD levels compared to cumulative doxorubicin doses of $<90 \mathrm{mg} / \mathrm{m}^{2}$. However, this outcome was not statistically significant, nor was it consistent with a study by Akreathy et al. ${ }^{11}$ which examined cardiotoxicity caused by doxorubicin in mice. They found that doxorubicin reduced total serum antioxidant levels, increasing cardiotoxicity $(\mathrm{P}<0.05)$. Furthermore, breast cancer patients treated with doxorubicin had reduced SOD levels $(\mathrm{P}<0.01) .{ }^{12}$ Decreased antioxidant levels are due to the doxorubicin sugar moiety, which has a ring attached to the tetracycline-containing quinone structure. Doxorubicin may compete with coenzyme Q10, which also has a quinone structure as an electron acceptor, resulting in free radical production. Doxorubicin can form semiquinone radical intermediates, which react with oxygen to produce superoxide anion radicals, leading to the production of hydrogen peroxide and hydroxyl radicals. ${ }^{13,14}$ Antioxidant such as SOD is needed to neutralize free radical. The more free radical is formed, the more antioxidant is used, finally can decrease antioxidant level.

Passive smokers had a hazard ratio of 0.96 (95\%CI:0.370 to 2.496; $\mathrm{P}=0.93$ ). This result shows that the risk of decreased SOD levels was 0.9 times lower in passive smokers than in children who were not exposed to passive smoking, but the result was not statistically significant. A study reported increased SOD levels in passive smokers compared to non-smokers $(\mathrm{P}>0.05) .{ }^{15}$ Tobacco smoke contains numerous compounds, many of which are oxidants and prooxidants, capable of producing free radicals and enhancing oxidative stress. Passive smokers have higher levels of oxidant.

Malnutrition had a hazard ratio of $1.06(95 \% \mathrm{CI}$ 0.094 to $11.864 ; \mathrm{P}=0.96$ ), suggesting a 1.06 times greater risk of decreased SOD levels compared to those with good nutrition, but the result was not statistically significant. Children with malnutrition have decreased antioxidant levels. Many factors may influence antioxidant levels, such as low serum zinc, vitamins A and $\mathrm{C}$, and other micronutrients in the body, leading 
to greater cellular sensitivity to oxidative stress and decreased SOD levels. ${ }^{16}$

Univariate analysis revealed that significantly more subjects who received a cumulative Mtx dose of $\geq 3000 \mathrm{mg} / \mathrm{m}^{2}$ had low SOD levels, compared to those with high SOD levels.

In conclusion, there is a significant relationship between higher cumulative doses of Mtx and decreased SOD levels. A cumulative Mtx dose of $\geq 3000 \mathrm{mg} / \mathrm{m}^{2}$ has a 9.96 times higher risk of decreased SOD levels compared to cumulative doses $<3000 \mathrm{mg} / \mathrm{m}^{2}$. There are no significant associations between higher cumulative doxorubicin dose, nutritional status, or passive smoking and decreased SOD levels.

\section{Conflict of interest}

None declared.

\section{References}

1. Mulatsih S, Sumadiono, Sutaryo, Purwanto. The result of treating children's acute lymphoblastic leukemia (ALL) in Dr. Sardjito Hospital with WK-LLA protocol 1999-2002. Buletin Ilmu Kesehatan Anak FK UNAIR. Tahun XXXI. 2004;17:808-19.

2. Al-Tonbary Y, Al-Haggar M, El-Ashry R, El-Dakroory S, Azzam H, Fouda A. Vitamin e and N-acetylcysteine as antioxidant adjuvant therapy in children with acute lymphoblastic leukemia. Adv Hematol. 2009;15:1-5.

3. Cetin T, Arpaci F, Yilmaz MI, Saglam K, Ozturk B, Komurcu $\mathrm{S}$, et al. Oxidative stress in patients undergoing high-dose chemotherapy plus peripheral blood stem cell transplantation. Biol Trace Elem Res. 2004;97:237-47.

4. Tavazzi B, Di Pierro D, Amorini AM, Fazzina G, Tuttobene M, Giardina B, et al. Energy metabolism and lipid peroxidation of human erythrocytes as a function of increased oxidative stress. Eur J Biochem. 2000;267:684-9.
5. Coleshowers CL, Oguntibeju OO, Ukpong M, Truter EJ. Effect of methotrexate on antioxidant enzyme status in a rodent model. Med Technol. 2010;24:5-9.

6. Esfahani A, Ghoreishi Z, Nikanfar A, Sanaat Z, Ghorbanihaghjo, A, Rashtchizaden N. Influence of chemotherapy on the oxidant/antioxidant status in patients with acute myeloid leukemia. IJHOSCR. 2009;3:3-4.

7. Gonzales R, Auclair C, Voisin E, Gautero H, Dhermy D, Boivin P. Superoxide dismutase, catalase, and glutathione peroxidase in red blood cells from patients with malignant disease. Cancer Res. 1984;44:4137-9.

8. Khan AU, Sheikh MU, Intekhab K. Pre-existing malnutrition and treatment outcome in children with acute lymphoblastic leukaemia. J Pak Med Assoc. 2006;56:171-3.

9. Asci H, Ozer MK, Calapoglu M, Savran M, Oncu M, Yesilot S, et al. Effects of misoprostol on methotrexate-induced hepatic and renal damages. J Biol Life Sci. 2011;2:32-7.

10. Devrim E, Cetin R, Kilicoglu B, Erguder B, Avci A, Durak I. Methotrexate causes oxidative stress in rat kidney tissues. Ren Fail. 2005;27:771-3.

11. Alkreathy H, Damanhouri ZA, Amhed N, Slevin M, Ali SS, Osman AM. Aged garlic extract protects against doxorubicin-induced cardiotoxicity in rats. Food Chem Toxicol. 2010;48:951-6.

12. Singh G, Maulik SK, Jaiswal A, Kumar P, Parshad R. Effect on antioxidant levels in patients of breast carcinoma during neoadjuvant chemotherapy and mastectomy. Malays J Med Sci. 2010;17:24-8.

13. Conklin KA. Chemotherapy-associated oxidative stress: impact on chemotherapeutic effectiveness. Integr Cancer Ther. 2004;3:294-300.

14. Tandon VR, Sharma S, Mahajan A, Bardi GH. Oxidative stress: a novel strategy in cancer treatment. JK Science. 2000;7:1-6.

15. Yokus B, Mete N, Cakir UD, Toprak G. Effect of active and passive smoking on antioxidant enzymes and antioxidant micronutrients. Biotechnol \& Biotechnol Eq. 2005;19:1-5.

16. Sharda B. Free radicals: emerging challenge in environmental health reseach in childhood and neonatal disorders. Int J Environ Res Public Health. 2006;3:286-91. 